\title{
Efeito do Tratamento Alcalino de Fibras de Curauá sobre as Propriedades de Compósitos de Matriz Biodegradável
}

\author{
Lílian Vanessa Rossa Beltrami, Lisete Cristine Scienza, Ademir José Zattera \\ Laboratório de Polímeros - LPOL, Centro de Ciências Exatas e Tecnologia - CCET, \\ Universidade de Caxias do Sul - UCS
}

\begin{abstract}
Resumo: Neste trabalho avaliou-se a influência do tratamento alcalino das fibras de curauá, nas concentrações de 1, 5 e $10 \%(\mathrm{~m} / \mathrm{v})$ de $\mathrm{NaOH}$, nas propriedades dos compósitos com matriz de PHBV e $20 \% \mathrm{~m} / \mathrm{m}$ de fibras de curauá. Os compósitos foram moldados por extrusão e injeção e avaliadas suas as propriedades morfológicas, térmicas e mecânicas. Os resultados de FTIR mostraram que o tratamento alcalino promoveu modificações na estrutura das fibras de curauá, comprovadas pela remoção da hemicelulose e da lignina, evidenciadas pela ausência das bandas típicas destes compostos. Nos compósitos com fibras de curauá tratadas com solução de $\mathrm{NaOH}$ na concentração de $5 \%$ apresentaram um aumento de $30 \%$ na resistência à flexão e $12 \%$ na resistência ao impacto, quando comparados aos compósitos com fibras sem tratamento. Estes resultados indicam que o tratamento alcalino promoveu uma melhoria na adesão das fibras na matriz, o que melhorou significativamente as propriedades mecânicas dos compósitos.
\end{abstract}

Palavras-chave: Compósitos, PHBV, fibras de curauá, tratamento alcalino.

\section{Effect of the Alkaline Treatments of Curauá Fiber on the Properties of Biodegradable Matrix Composites}

\begin{abstract}
This study investigate the influence of alkaline treatment applied to Curauá fibers, at concentrations of 1,5 and $10 \%$ (w/v) $\mathrm{NaOH}$, on the properties of the composite of PHBV matrix with $20 \mathrm{wt} \%$ Curauá fiber. The composites were molded by extrusion and injection and their morphological, thermal and mechanical properties were evaluated. The results showed that alkali treatment caused modifications in the structure of the Curauá fibers by the removal of hemicellulose and lignin, as evidenced by the absence of the typical bands of these compounds in FTIR analysis. It was found an increase of $30 \%$ in flexural strength and $12 \%$ in impact resistance for the composites with fibers treated in $5 \% \mathrm{NaOH}$ solution, in relation to composites with fibers without treatment. These results indicate that the alkaline treatment promoted an improvement in the adhesion of the fibers to the matrix, which significantly improved the mechanical properties of the composites. However, concentrations of $\mathrm{NaOH}$ solution different from $5 \%$ may have a deleterious effect on the mechanical properties of the fibers and composites.
\end{abstract}

Keywords: Composite, PHBV, curaua fibers, alkaline treatments.

\section{Introdução}

Uma tendência mundial crescente para a utilização máxima dos recursos naturais através de novos processos e produtos tem conduzido ao estudo e exploração de materiais provenientes de fontes naturais renováveis ${ }^{[1]}$ e, dentro deste contexto, os compósitos biodegradáveis têm ganho relevância considerável no desenvolvimento de novos materiais ${ }^{[2]}$.

Compósitos com fibras naturais têm propriedades mecânicas superiores ao polímero puro $^{[3-5]}$ e que a capacidade de processamento dos compósitos reforçados com fibras vegetais está diretamente relacionada ao índice de fluidez da matriz e ao teor de fibras utilizado ${ }^{[6]}$. Dentre as fibras vegetais utilizadas como reforço em compósitos estão as fibras de curauá. O curauá (Ananas erectifolius L.B. Smith), uma bromélia característica da Amazônia paraense muito utilizadas na indústria devido a sua resistência, maciez, leveza e reciclabilidade ${ }^{[7]}$.

As fibras de curauá têm alta resistência mecânica quando comparadas com outras fibras como juta, sisal e linho. Possuem propriedades mecânicas semelhantes às das fibras de vidro, como rigidez, resistência ao impacto e flexibilidade ${ }^{[8,9]}$. Outras propriedades que as fibras vegetais apresentam incluem baixa abrasão no equipamento e menor consumo de energia ${ }^{[10]}$.

Um polímero é considerado biodegradável se todos os seus componentes orgânicos sofrem uma biodegradação total $^{[11]}$. A biodegradação ocorre quando o polímero é usado como nutriente por um determinado grupo de microrganismos (bactérias, fungos ou algas) que coexistem no meio ambiente, convertendo-o a compostos mais simples, sendo mineralizados e redistribuídos por meio de ciclos elementares, tais como o do carbono, do nitrogênio e do enxofre ${ }^{[12]}$.

Os polímeros da família dos polihidroxialcanoatos (PHAs), além de biodegradáveis, utilizam de recursos renováveis para sua produção ${ }^{[13]}$. Entre os PHAs mais pesquisados está poli(hidroxibutiratoco-hidroxivalerato) - $\mathrm{PHBV}^{[14-16]}$. O PHBV é um 
polímero semi-cristalino com propriedades mecânicas e térmicas semelhantes as do polipropileno $(\mathrm{PP})^{[17,18]} \mathrm{e}$ estas características o tornam um potencial substituto para resinas de petróleo, com aplicações na indústria automotiva e aplicações biomédicas, como válvulas cardíacas e produtos ortopédicos ${ }^{[13,18]}$.

A interação entre os componentes de um compósito está centrada nas características da interface entre eles, a qual deve permitir uma transferência eficiente de tensão da matriz para o reforço. Um dos efeitos que contribuem para a adesão na interface é a atração física entre átomos polares da matriz e do reforço. Problemas de adesão entre a fibra e a matriz polimérica podem diminuir a transferência de esforços, comprometendo as propriedades mecânicas do compósito ${ }^{[19]}$. Assim, para promover uma maior adesão, as fibras são submetidas a um pré-tratamento superficial apropriado e/ou um agente de acoplamento é adicionado ao compósito.

$\mathrm{O}$ tratamento alcalino, normalmente realizado em solução aquosa de hidróxido de sódio $(\mathrm{NaOH})$, é um dos tratamentos químicos mais usados para as diversas fibras vegetais. A modificação ocasionada por esse tratamento é o rompimento da ligação de hidrogênio no grupo hidroxila $(\mathrm{OH})$ presente na estrutura da fibra, aumentando assim a rugosidade superficial ${ }^{[20,21]}$. Este tratamento remove certa quantidade de lignina, cera e óleos que recobrem a superfície externa da parede celular da fibra, despolimerizando a celulose amorfa, removendo grande parte da hemicelulose das fibras. A adição de solução aquosa de $\mathrm{NaOH}$ na fibra promove a ionização do grupo $\mathrm{OH}$ a alcóxido ${ }^{[22]}$. Embora esse tratamento aumente a adesão com uma matriz polimérica, a resistência mecânica da fibra pode ser significativamente reduzida dependendo da concentração da solução alcalina ${ }^{[20,22]}$, comprometendo a resistência do compósito fabricado com estas fibras.

Estudos de Campos et al. ${ }^{[23]}$ mostraram que os compósitos com matriz termoplástica e fibras de sisal tratadas com $\mathrm{NaOH}$ apresentaram melhora na resistência à tração e na estabilidade térmica, o que indica uma melhor adesão fibra/matriz, quando comparados com compósitos de fibras de sisal submetidas à outros tratamento.

A pesquisa realizada por Pires et al..$^{[24]}$ sobre o efeito do tratamento alcalino de fibras de juta no comportamento mecânico de compósitos de matriz epóxi mostrou que a modificação química das fibras de juta com tratamento alcalino foi evidenciada pela técnica de infravermelho, devido à remoção da hemicelulose. Os compósitos com fibras tratadas apresentaram aumento nas propriedades mecânicas em relação à resina sem reforço e aos compósitos com fibras sem tratamento.

Dentro deste contexto, este trabalho teve como objetivo avaliar o efeito do tratamento alcalino em fibras de curauá na morfologia e nas propriedades térmicas e mecânicas de compósitos de matriz de PHBV.

\section{Experimental}

Foram empregados o polímero PHBV em grânulos, produzido pela PHB Industrial, contendo $18,25 \%(\mathrm{~m} / \mathrm{m})$ de hidroxivalerato (HV); as fibras de curauá (FCur), provenientes do município de Santarém, no Pará, foram compradas do Centro de Apoio a Projetos de Ação
Comunitária (CEAPAC) e o hidróxido de sódio P.A., da marca Sigma-Aldrich.

\section{Preparação das fibras}

As fibras de curauá foram recebidas em forma de fibras longas, pós-colheita e secagem, sem nenhum tratamento prévio. As fibras foram penteadas para a remoção das cascas e das sujidades de sua superfície e cortadas em $2,5 \mathrm{~cm}$ de comprimento. Aplicou-se um tratamento alcalino, variando-se as concentrações de $\mathrm{NaOH}$ na solução em 1,5 e $10 \%(\mathrm{~m} / \mathrm{v})$. As fibras permaneceram imersas na solução de $\mathrm{NaOH}$, na proporção de 10:1 (solução:fibras), por 2 horas a temperatura de $50{ }^{\circ} \mathrm{C}$. As fibras foram lavadas com água destilada até que a solução de lavagem remanescente apresentasse $\mathrm{pH}$ 7,0 e, posteriormente, as fibras foram secas em estufa a uma temperatura de $60{ }^{\circ} \mathrm{C}$ por 12 horas.

\section{Preparação dos compósitos}

O polímero e as fibras foram processados em uma extrusora monorrosca (modelo ES 35FR, marca SEIBT), com perfil de temperaturas variando de 150 a $160{ }^{\circ} \mathrm{C}$ e rotação de $50 \mathrm{rpm}$, a fim de promover uma pré-mistura e facilitar as demais etapas do processamento. $\mathrm{O}$ material extrudado foi moído em moinho de facas (Primotécnica, modelo 1001) e submetido a uma segunda extrusão em extrusora dupla-rosca co-rotacional (MH Equipamentos, modelo COR 20-32-LAB), com 8 zonas de aquecimento e perfil de temperaturas variando de 150 a $165{ }^{\circ} \mathrm{C}$, rotação de $200 \mathrm{rpm}$, com rosca de diâmetro de $20 \mathrm{~mm}$ e $\mathrm{L} / \mathrm{D}=32$. O material foi novamente moído, sendo o pó obtido posteriormente moldado por injeção (Himaco Hidráulicos e Máquinas Ltda., modelo LHS 150-80) com perfil de temperaturas de 145,155 e $160{ }^{\circ} \mathrm{C}$ e velocidade da rosca de $100 \mathrm{rpm}$. Antes de cada etapa do processo o material foi mantido por 24 horas em estufa a $60{ }^{\circ} \mathrm{C}$, para a remoção da umidade.

Foi definida a concentração de $20 \%(\mathrm{~m} / \mathrm{m})$ de fibras para os compósitos, tendo o como base os resultados do estudo realizado por Rossa et al. ${ }^{[25]}$.

\section{Caracterização}

A morfologia superficial das fibras de curauá e a superfície de fratura dos compósitos foram investigadas empregando um microscópio eletrônico de varredura Shimadzu SSX-550 Superscan, com uma tensão de aceleração de $10 \mathrm{kV}$.

Os espectros de infravermelho das fibras de curauá foram obtidos em um espectrofotômetro Nicolet IS10 Termo Scientific, modo ATR, na faixa de $4000 \mathrm{~cm}^{-1}$ e $400 \mathrm{~cm}^{-1}$.

A temperatura de degradação dos compósitos foi avaliada empregando-se a técnica de termogravimetria utilizando-se um equipamento Shimadzu TGA-50, na faixa de temperatura de $23{ }^{\circ} \mathrm{C}$ a $700{ }^{\circ} \mathrm{C}$, com uma taxa de aquecimento de $10{ }^{\circ} \mathrm{C} \cdot \mathrm{min}^{-1}$ em atmosfera de $\mathrm{N}_{2}$ com fluxo de $50 \mathrm{~mL} \cdot \mathrm{min}^{-1}$. A massa utilizada foi de aproximadamente $10 \mathrm{mg}$ do material moído criogênicamente. 
Os ensaios mecânicos de resistência à tração e à flexão foram realizados nos compósitos empregando-se uma máquina universal de ensaio EMIC DL 2000, em conformidade com as normas ASTM D638-10 e ASTM D790-10, respectivamente. No ensaio de resistência à tração utilizou-se uma célula de carga de $2.000 \mathrm{kgf}$ e velocidade de $5 \mathrm{~mm} \cdot \mathrm{min}^{-1} \mathrm{e}$ no ensaio de resistência à flexão utilizando-se uma célula de carga de $100 \mathrm{kgf}$ e velocidade de $1,5 \mathrm{~mm} \cdot \mathrm{min}^{-1}$.

O ensaio de resistência ao impacto IZOD, sem entalhe, com pêndulo de 2,75 J foi realizado em equipamento da CEAST, modelo Resil 25, conforme a norma ASTM D256-10. As dimensões dos corpos de prova utilizados estão em conformidade com as normas citadas, sendo que, para cada ensaio utilizou-se 5 corpos de prova, que permaneceram por 48 horas em um ambiente fechado com temperatura de $23{ }^{\circ} \mathrm{C}\left( \pm 2{ }^{\circ} \mathrm{C}\right)$ e umidade relativa de $50 \%( \pm 10 \%)$

\section{Resultados e Discussões}

Na Figura 1 observa-se as micrografias das fibras de curauá sem e como tratamento alcalino com diferentes concentrações de $\mathrm{NaOH}$. Nota-se que quanto mais intenso o tratamento alcalino maior a remoção dos componentes amorfos da superfície da fibra, expondo as microfibrilas, promovendo um aumento da rugosidade da superfície e facilitando a desfibrilação.

Estudos de Hossain et al. ${ }^{[26]}$ mostraram que o tratamento alcalino em fibras de juta promoveu a desfibrilação e o aumento da rugosidade da superfície das fibras, sendo estas mudanças atribuídas à remoção de impurezas, de componentes não-celulósicos e de ceras. Segundo os autores, o tratamento alcalino resulta no aumento de ligações $\mathrm{OH}$ disponíveis na superfície e na desfibrilação das fibras, o que aumenta a área superficial de contato da fibra com a matriz, melhorando a interação entre elas, quando as matrizes são polares.
Na Figura 2 observa-se as micrografias dos compósitos PHBV/FCur sem e com tratamento alcalino em diferentes concentrações de $\mathrm{NaOH}$. Nota-se a presença de vazio na interface entre as fibras e a matriz polimérica, sugerindo que o tratamento alcalino não promoveu diretamente uma maior adesão entre as fibras e a matriz.

Resultado semelhante foi observado por Spinacé et al. ${ }^{[27]}$ em seu estudo sobre compósitos de polipropileno reciclado reforçado com fibras de curauá. Os autores relataram que a adesão da fibra na matriz polimérica não foi melhorada com o tratamento alcalino, pois pelas micrografias foi possível observar que as fibras foram facilmente arrancadas da matriz (pull-out).

A fraca interação entre a fibra e a matriz está relacionada à energia de superfície, pois as fibras em geral são predominantemente polares, enquanto as matrizes poliméricas são apolares ${ }^{[28]}$. Segundo Paul et al. ${ }^{[29]}$ a adição de um agente de acoplamento promoveria uma melhora na adesão entre as partes, pois este reduz a acidez superficial da fibra, tornando-a menos polar e assim mais compatível com a matriz polimérica.

A Figura 3 apresenta os espectros de infravermelho das fibras de curauá sem e com tratamento alcalino. Observa-se nas fibras tratadas uma diminuição na intensidade na banda larga em $3400-3300 \mathrm{~cm}^{-1}$, referente às ligações $\mathrm{O}-\mathrm{H}$ da celulose e da água.

A dissolução da hemicelulose na solução alcalina é comprovada através da redução da intensidade banda em torno de $2915 \mathrm{~cm}^{-1} \mathrm{e}$ da ausência da banda em $1730 \mathrm{~cm}^{-1}$, que correspondem, respectivamente, às ligações $\mathrm{C}-\mathrm{H}$ e $\mathrm{C}=\mathrm{O}$ do grupo éter da hemicelulose.

A remoção da lignina é observada nas fibras tratadas, sendo indicada pela ausência da banda em $1512 \mathrm{~cm}^{-1}$, pela diminuição da intensidade da banda em $1450 \mathrm{~cm}^{-1} \mathrm{e}$ pela ausência da banda em $1245 \mathrm{~cm}^{-1}$, correspondentes, à vibração do anel benzênico e aos estiramentos das ligações C-H e C-O do grupo acetila da lignina, respectivamente.

Estudos realizados por Hossain ${ }^{[26]}$ e por Ibrahim et al. ${ }^{[30]}$, que empregaram tratamento alcalino em

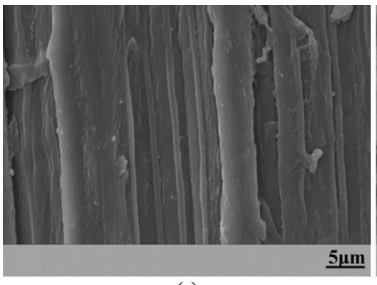

(a)

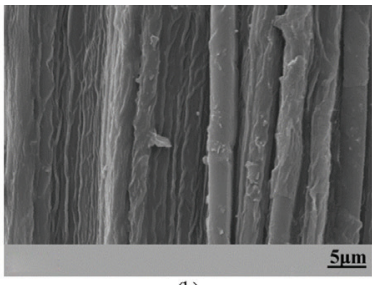

(b)

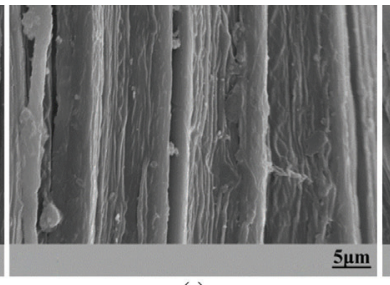

(c)

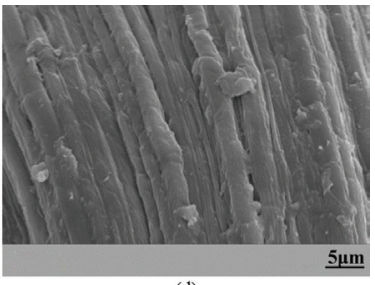

(d)

Figura 1. Micrografias de MEV da FCur: (a) sem tratamento e tratadas com solução de (b) $1 \% \mathrm{NaOH}$; (c) $5 \% \mathrm{NaOH}$ e (d) $10 \% \mathrm{NaOH}$, com magnitude de 2000x.

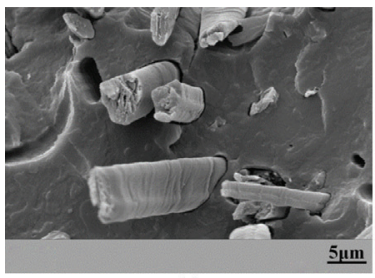

(a)

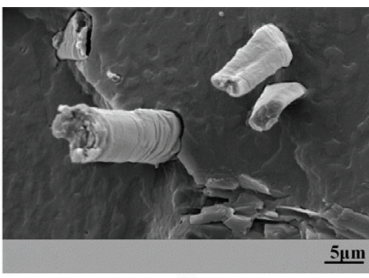

(b)

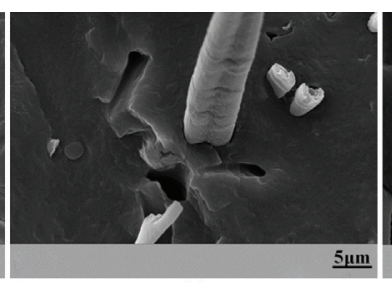

(c)

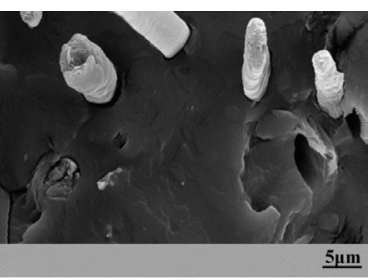

(d)

Figura 2. Micrografias por MEV dos compósitos com FCur: (a) sem tratamento e tratadas com solução de (b) $1 \% \mathrm{NaOH}$; (c) $5 \% \mathrm{NaOH}$ e (d) $10 \% \mathrm{NaOH}$, com magnitude de 2000x. 
fibras vegetais, estão em conformidade com os resultados encontrados no presente estudo e confirmam que este tipo de tratamento remove a hemicelulose e a lignina das superfícies das fibras.

A mudança na composição química das fibras de curauá tratadas em relação a sem tratamento acarretou uma diminuição na estabilidade térmica de mais de $10{ }^{\circ} \mathrm{C}$, conforme observado na Figura 4. Esta redução na temperatura de degradação térmica é atribuída à remoção da lignina, que pirolisa a temperaturas mais altas do que a celulose e também à conversão de parte da celulose I em celulose II.

Segundo Klemm et al. ${ }^{[31]}$ com o tratamento alcalino, em condições específicas de concentração, temperatura e agitação, a celulose expande-se e suas cadeias são rearranjadas, alterando a estrutura cristalina da celulose, convertendo-a de celulose I para celulose II, termicamente mais estável.

A redução na temperatura de degradação de fibras vegetais também foi constatada por Gurgel ${ }^{[32]}$, que relacionou este fato à conversão incompleta de celulose I em celulose II. Segundo o autor, é necessário um tratamento alcalino adicional, o qual removeria o teor de lignina residual e proporcionaria a completa modificação

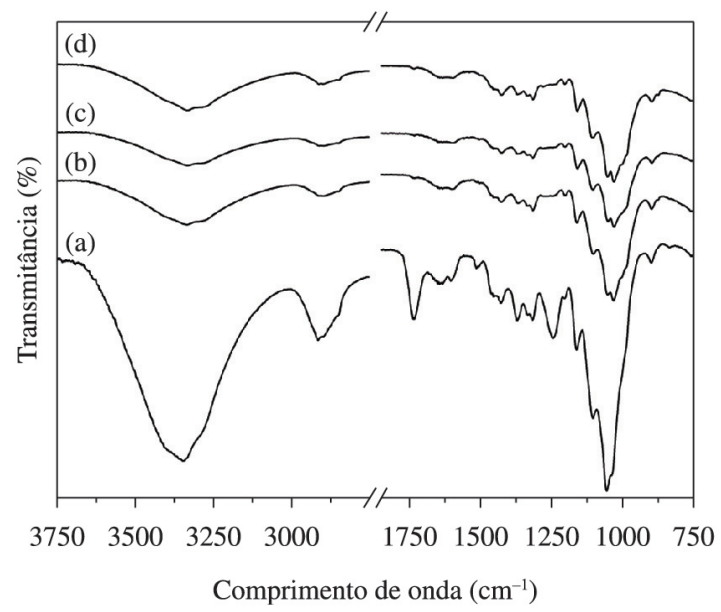

Figura 3. FTIR-ATR da FCur: (a) sem tratamento e tratadas com solução de (b) $1 \% \mathrm{NaOH}$; (c) $5 \% \mathrm{NaOH}$ e (d) $10 \% \mathrm{NaOH}$.

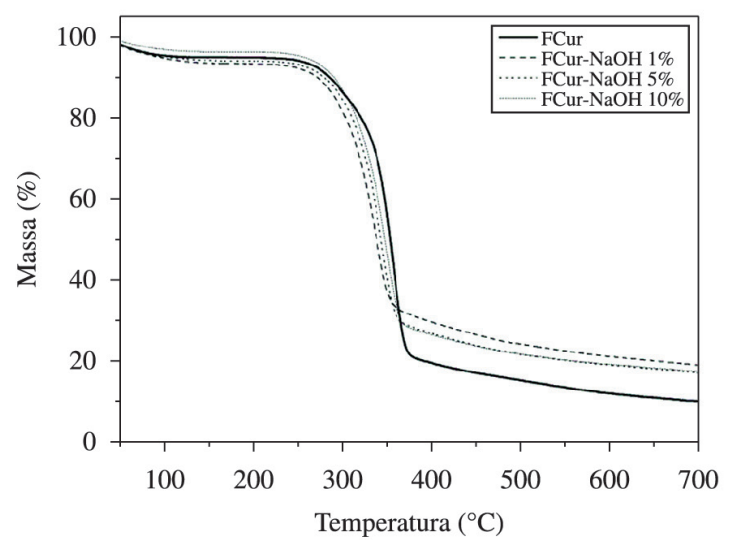

para celulose II, responsável pelo aumento da estabilidade térmica da fibra.

As alterações térmicas provocadas pelo tratamento alcalino nas fibras são também observadas nos compósitos (Figura 5). A Tabela 1 apresenta as caraterísticas térmicas dos compósitos com fibras de curauá tratadas com solução de $\mathrm{NaOH}$ em diferentes concentrações.

Gutiérrezetal. ${ }^{[33]}$ observaramomesmocomportamento térmico em compósitos com fibras de curauá tratadas e, segundo os autores, as propriedades térmicas dos compósitos são determinadas, principalmente, pelas propriedades das fibras utilizadas, o qual depende do tratamento efetuado.

Observa-se na Figura 6 que a resistência à tração dos compósitos aumentou em 3\% para os compósitos com fibra tratada com $1 \%$ e $5 \%$ de $\mathrm{NaOH}$ e diminuiu em $2 \%$ para os compósitos com fibra tratada com $10 \%$ de $\mathrm{NaOH}$. Considerando-se o erro experimental do ensaio, pode-se considerar que os resultados não apresentaram modificações significativas.

A resistência à flexão dos compósitos aumentou em $8 \%$ e $30 \%$ para os compósitos com fibra tratada com solução com $1 \%$ e $5 \%$ de $\mathrm{NaOH}$, respectivamente, e não sofreu alterações nos compósitos com fibra tratada com solução com $10 \%$ de $\mathrm{NaOH}$.

Observa-se que o módulo de elasticidade de resistência à tração aumentou em $24 \%$ para os compósitos com fibra tratada com solução com $5 \%$ de $\mathrm{NaOH}$, enquanto que para os demais compósitos não apresentou alteração apreciáveis. Avaliando-se o módulo de elasticidade de resistência à flexão, observa-se que os compósitos com fibras tratadas não sofreram alterações significativas quando comparado ao polímero puro.

Observou-se que a deformação na ruptura para todas os compósitos não teve variação apreciável, permanecendo em torno de $4 \%$. O módulo de elasticidade bem como a deformação na ruptura depende principalmente da eficiência de transferência de tensões da matriz para as fibras, o que depende da magnitude das interações na interface fibra-matriz.

Observa-se na Figura 7 que a resistência ao impacto dos compósitos diminui em $2 \%$ para os compósitos com fibra tratada com $1 \% \mathrm{NaOH}$ e aumenta em $12 \%$ e $3 \%$ para os compósitos com fibra tratada com $5 \%$ e $10 \%$ de $\mathrm{NaOH}$, respectivamente.

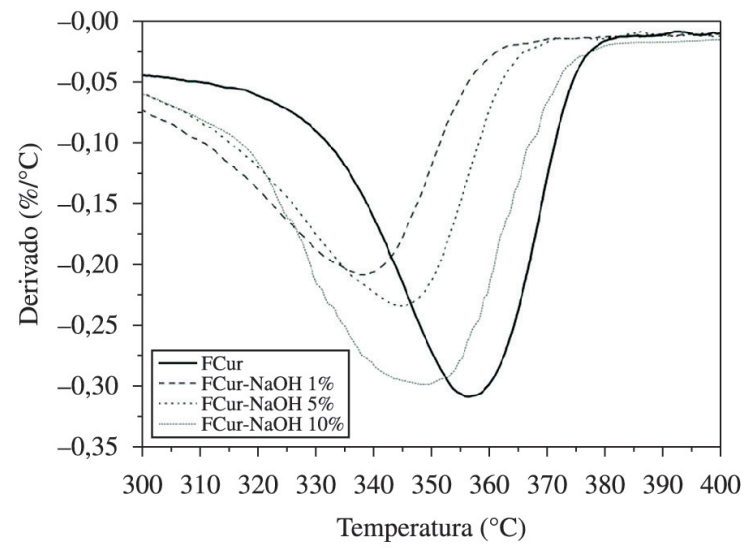

Figura 4. Termogramas de TGA e DTGA para as fibras de curauá sem e com tratamento alcalino. 


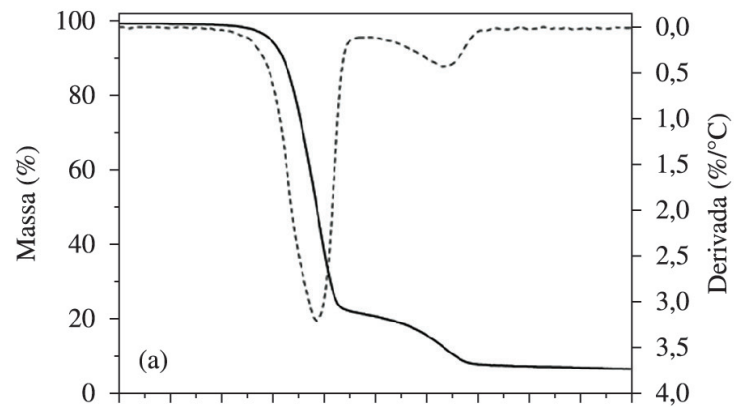

200225250275300325350375400425450

Temperatura $\left({ }^{\circ} \mathrm{C}\right)$

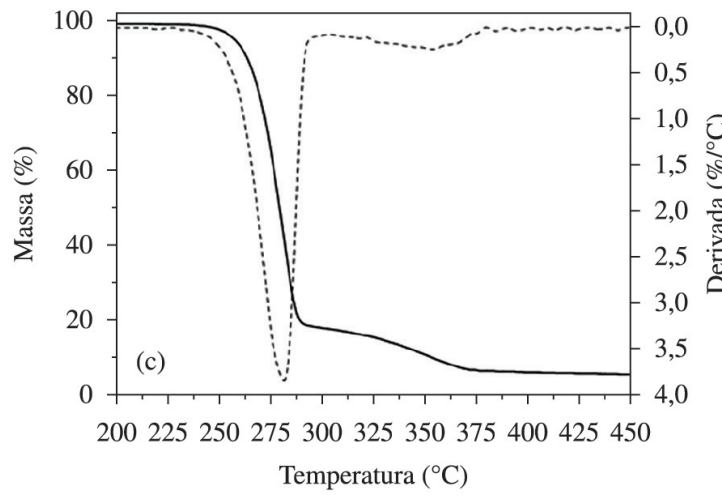

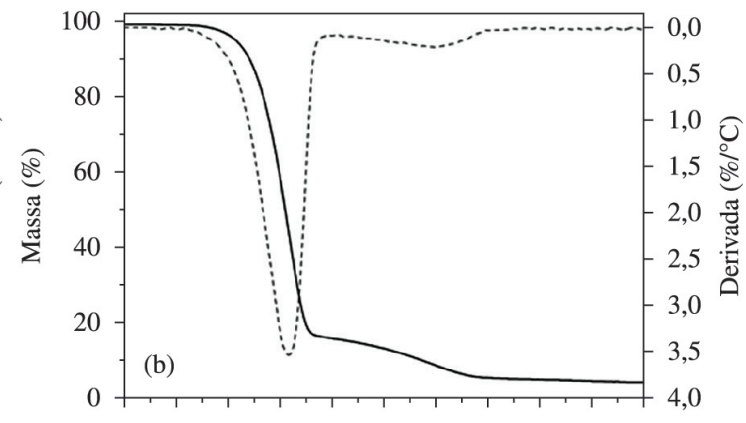

200225250275300325350375400425450

Temperatura $\left({ }^{\circ} \mathrm{C}\right)$

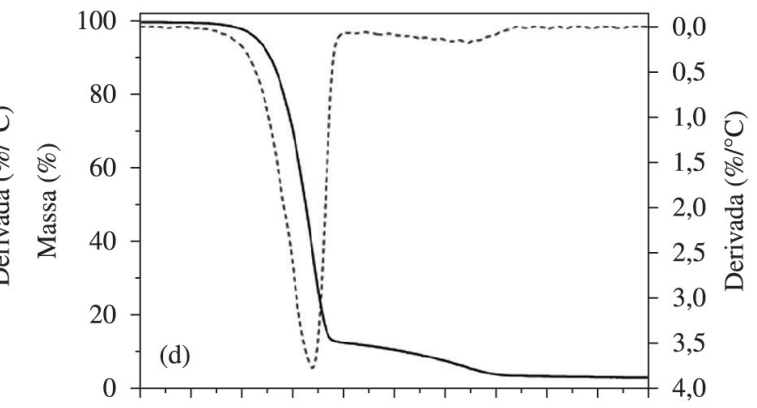

200225250275300325350375400425450

Temperatura $\left({ }^{\circ} \mathrm{C}\right)$

Figura 5. Termogramas de TGA e DTGA para os compósitos com FCur: (a) sem tratamento e tratadas com solução de (b) $1 \%$ NaOH; (c) $5 \% \mathrm{NaOH}$ e (d) $10 \% \mathrm{NaOH}$.

Tabela 1. Características térmicas dos compósitos de PHBV/FCur tratadas com solução de NaOH em diferentes concentrações.

\begin{tabular}{lccccc}
\hline \multicolumn{1}{c}{ Amostra } & Degradação & $\mathbf{T}_{\text {onset }}\left({ }^{\circ} \mathbf{C}\right)$ & $\mathbf{T}_{\text {max }}\left({ }^{\circ} \mathbf{C}\right)$ & $\mathbf{T}_{\text {endset }}\left({ }^{\circ} \mathbf{C}\right)$ & Perda de massa $(\boldsymbol{\%})$ \\
\hline PHBV/FCur & $1^{\circ}$ & 276 & 296 & 305 & 75 \\
sem tratamento & $2^{\circ}$ & 320 & 359 & 366 & 95 \\
PHBV/FCur & $1^{\circ}$ & 260 & 279 & 287 & 88 \\
$1 \% \mathrm{NaOH}$ & $2^{\circ}$ & 305 & 348 & 357 & 98 \\
$\mathrm{PHBV} / \mathrm{FCur}$ & $1^{\circ}$ & 260 & 280 & 287 & 88 \\
$5 \% \mathrm{NaOH}$ & $2^{\circ}$ & 302 & 348 & 360 & 96 \\
$\mathrm{PHBV} / \mathrm{FCur}$ & $1^{\circ}$ & 261 & 350 & 288 & 89 \\
$10 \% \mathrm{NaOH}$ & $2^{\circ}$ & 307 & & 361 & 98 \\
\hline
\end{tabular}

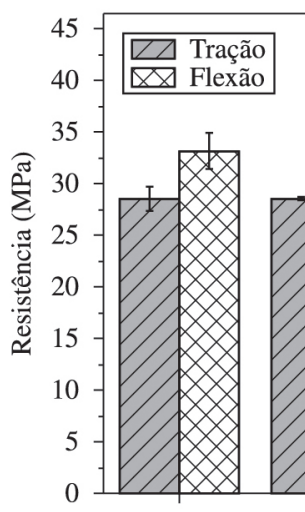

(a)

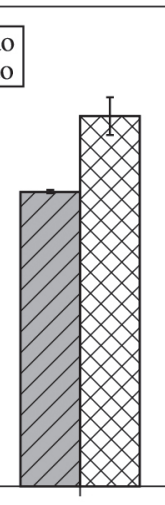

(b)

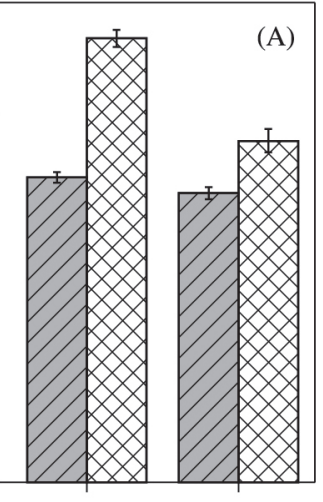

(c)

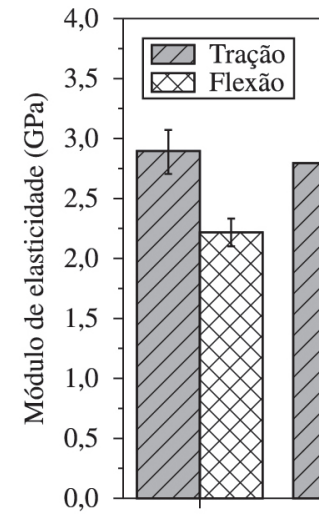

(a)

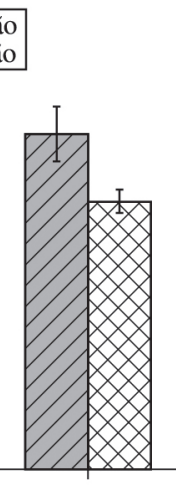

(b)

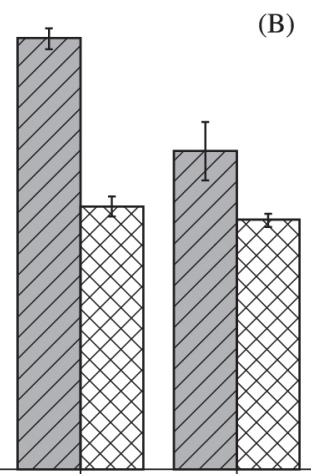

(c)

(d)

Figura 6. Resistência à tração e à flexão (A) e módulos de elasticidade (B) dos compósitos com FCur: (a) sem tratamento e tratadas com solução de (b) $1 \% \mathrm{NaOH}$; (c) $5 \% \mathrm{NaOH}$ e (d) $10 \% \mathrm{NaOH}$. 


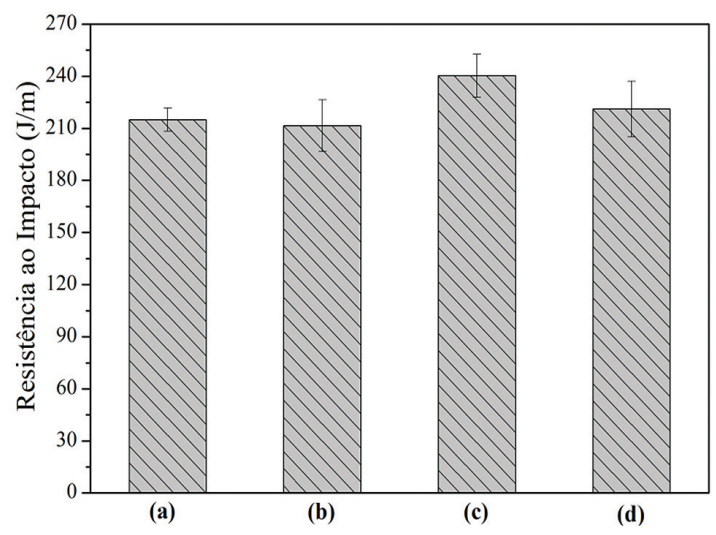

Figura 7. Resistência ao impacto dos compósitos com FCur: (a) sem tratamento e tratadas com solução de (b) $1 \% \mathrm{NaOH}$; (c) $5 \% \mathrm{NaOH}$ e (d) $10 \% \mathrm{NaOH}$.

Resutados semelhantes aos observados foram relatados no estudo de Ahankari et al. ${ }^{[34]}$, que mostrou que os compósitos de PHBV reforçado com palha de trigo expostas ao tratamento alcalino não tiveram alterações significativas na resistência e no módulo de elasticidade de tração. Contudo, as resistências à flexão e ao impacto aumentaram cerca de $10 \%$ e $35 \%$, respectivamente, quando comparadas ao compósito com fibra não tratada. Estas melhorias nas propriedades são consequência do aumento de área superficial de contado da fibra com a matriz devido à desfibrilação promovida cisalhamento ocorrido durante o processo de extrusão e favorecida pelo tratamento alcalino. De acordo com Pothan et al. ${ }^{[35]}$, além da desfibrilação, o tratamento alcalino promove a diminuiu sua acidez da superfície da fibra, devido a presença de grupos $\mathrm{OH}$, contribuindo para o aumento da interação polar-polar entre a matriz e a fibra.

\section{Conclusões}

O tratamento alcalino promoveu modificações químicas nas fibras de curauá, comprovadas pela ausência das bandas típicas da hemicelulose e da lignina no ensaio de FTIR. Constatou-se que o tratamento alcalino reduziu a estabilidade térmica das fibras de curauá e, consequentemente, dos compósitos PHBV/FCur, indicando que o ataque químico converteu parcialmente a celulose.

Os compósitos com fibras tratadas com solução de $5 \% \mathrm{NaOH}$ apresentaram aumento de $3 \%$ na resistência à tração, 24\% no módulo de elasticidade de tração, 30\% na resistência à flexão e $12 \%$ na resistência ao impacto, quando comparados aos compósitos com fibras sem tratamento.

Com base nos resultados apresentados, observa-se que o tratamento realizado nas fibras de curauá com solução alcalina em baixa concentração $(1 \% \mathrm{NaOH})$ não promoveu uma melhora efetiva na adesão da fibra na matriz, não alterando significativamente as propriedades mecânicas dos compósitos. Por outo lado, o tratamento com solução alcalina em alta concentração (10\% $\mathrm{NaOH}$ ) fragilizou a estrutura das fibras, o que diminui as propriedades mecânicas dos compósitos. Logo, concluiu-se que o tratamento alcalino com solução de $5 \% \mathrm{NaOH}$ é o mais indicado à ser realizado sobre as fibras de curauá, pois promove a melhora na adesão da fibra na matriz polimérica, proporcionando um melhor desempenho mecânico dos compósitos.

\section{Agradecimentos}

Os autores agradecem à Prefeitura Municipal de Caxias do Sul para apoio financeiro e à Universidade de Caxias do Sul pelo apoio à pesquisa.

\section{Referências Bibliográficas}

1. John, M. J. \& Thomas, S. - Carbohyd. Polym., 71, p.343 (2008). http://dx.doi.org/10.1016/j.carbpol.2007.05.040

2. Neto, L. F. \& Pardini, L. C. - "Compósitos estruturais: ciência e tecnologia”, Edgard Blücher, São Paulo (2006).

3. Borsoi, C.; Scienza, L. C.; Zattera, A. J. \& Angrizani, C. C. - Polímeros, 21, p.271 (2011). http://dx.doi.org/10.1590/ S0104-14282011005000055

4. Poletto, M.; Dettenborn, J.; Zeni, M. \& Zattera, A. J. - Waste Manag., 31, p.779 (2011). PMid:21172732. http://dx.doi. org/10.1016/j.wasman.2010.10.027

5. Srubar III, W. V.; Pilla, S.; Wright, Z. C.; Ryan, C. A.; Greene J. P.; Frank, C. W. \& Billington, S. L. - Compos. Sci. Technol., 72, p.708 (2012). http://dx.doi.org/10.1016/j. compscitech.2012.01.021

6. Mano, B.; Araújo, J. R.; Spinacé, M. A. S. \& De Paoli, M. A. - Compos. Sci. Technol., 70, p.29 (2010). http://dx.doi. org/10.1016/j.compscitech.2009.09.002

7. Gomes, A.; Matsuo, T.; Goda, K. \& Ohgi, J. - Compos. Appl. Sci. Manuf., 38, p.1811 (2007). http://dx.doi. org/10.1016/j.compositesa.2007.04.010

8. Silva, R. V. \& Aquino E. M. F. - J. Reinf. Plast. Comp., 27, p.103 (2008). http://dx.doi.org/10.1177/073168440802700 10301

9. Amarasekera, J.; Santos, P. A.; Giriolli, J. C. \& Moraes, G. - Plast. Addit. Comp., 11, p.12 (2009).

10. Araújo, J. R.; Waldman, W. R. \& De Paoli, M. A. - Polym. Degrad. Stabil., 93, p.1770 (2008). http://dx.doi. org/10.1016/j.polymdegradstab.2008.07.021

11. De Paoli, M. A. - "Degradação e estabilização de polímeros". Ebah (2008). Disponível em: <http://www. ebah.com.br/degradacao-e-estabilizacao-de-polimerospdf-a35407.html>.

12. Vilaplana, F.; Strömberg, E. \& Karlsson, S. - Polym. Degrad. Stabil., 95, p. 2147 (2010). http://dx.doi.org/10.1016/j. polymdegradstab.2010.07.016

13. Javadi,A.; Srithep, Y.; Lee, J.; Pilla, S.; Clemons, C.; Gong, S. \& Turng, L. - Compos. Appl. Sci. Manuf., 41, p.982 (2010). http://dx.doi.org/10.1016/j.compositesa.2010.04.002

14. Villano, M.; Beccari, M.; Dionisi, D.; Lampis, S.; Miccheli, A.; Vallini, G. \& Majone, M. - Process. Biochem., 45, p.714 (2010). http://dx.doi.org/10.1016/j.procbio.2010.01.008

15. Liu, Z.; Wang, Y.; He, N.; Huang, J.; Zhu, K.; Shao, W.; Wang, H.; Yuan, W. \& Li, Q. - J. Hazard. Mater., 185, p.8 (2011). PMid:20970922. http://dx.doi.org/10.1016/j. jhazmat.2010.08.003 
16. Pivsa-Art, S.; Srisawat, N. \& O-Charoen, N. - Energy Procedia, 9, p.589 (2011). http://dx.doi.org/10.1016/j. egypro.2011.09.069

17. Martelli, S. M.; Sabirova, J. \& Fakhoury, F. M. - LWT - Food Sci. Technol., 47, p.386 (2012).

18. Lucchesi, C.; Ferreira, B. M. P.; Duek, E. A. R.; Santos Junior, A. R. \& Joazeiro, P. P. - J. Mater. Sci. Mater. Med., 19, p.635 (2008). PMid:17619989. http://dx.doi. org/10.1007/s10856-007-0169-3

19. Sreekumar, P. A.; Thomas, S. P.; Saiter, J. M.; Joseph, K.; Unnikrishnan, G. \& Thomas, S. - Compos. Appl. Sci. Manuf., 40, p.1777 (2009). http://dx.doi.org/10.1016/j. compositesa.2009.08.013

20. Amico, S. C.; Sydenstricker, T. H. D. \& Silva, P. S. C. P. - Met. Mater. Process., 17, p.233 (2005).

21. Sghaier, A. O. B.; Chaabouni, Y.; Msahli, S. \& Sakli, F. - Ind. Crop. Prod., 36, p.257 (2012).

22. Li, X.; Tabil, L. G. \& Panigrahi, S. - J. Polym. Environment, 15, p.25 (2007). http://dx.doi.org/10.1007/ s10924-006-0042-3

23. Campos, A.; Teodoro, K. B. R.; Marconcini, J. M.; Mattoso, L. H.C. \& Martins-Franchetti, S. M. - Polímeros, 21, p.217 (2011). http://dx.doi.org/10.1590/S0104-14282011005000039

24. Pires, E. N.; Merlini, C.; Al-Qureshi, H. A.; Salmória, G. V. \& Barra, G. M. O. - Polímeros, 22, p.339 (2012). http:// dx.doi.org/10.1590/S0104-14282012005000053

25. Rossa, L. V.; Scienza, L. C. \& Zattera, A. J. - Polym. Compos., 34, p.450 (2013). http://dx.doi.org/10.1002/ pc. 22432

26. Hossain, M. K.; Dewan, M. W.; Hosur, M. \& Jeelani, S. - Compos. Part B, 42, p.1701 (2011). http://dx.doi. org/10.1016/j.compositesb.2011.03.010
27. Spinacé, M. A. S.; Fermoseli, K. K. G. \& De Paoli, M. A. - J. Appl. Polym. Sci., 112, p.3686 (2009). http://dx.doi. org/10.1002/app.29683

28. Borsoi, C.; Scienza, L. C. \& Zattera, A. J. - J. Appl. Polym. Sci., 128, p.653 (2012). http://dx.doi.org/10.1002/ app.38236

29. Paul, S. A.; Joseph, K.; Gem Mathew, G. D.; Pothen, L. A. \& Thomas, S. - Compos. Appl. Sci. Manuf., 41, p.1380 (2010). http://dx.doi.org/10.1016/j.compositesa.2010.04.015

30. Ibrahim, N. A.; Hadithon, K. A. \& Abdan, K. - J. Reinf. Plast. Comp., 14, p.2192 (2010).

31. Klemm, D.; Heublein, B.; Fink, H. P. \& Bohn, A. - Angew. Chem. Int. Ed. Engl., 44, p.3358 (2005). PMid:15861454. http://dx.doi.org/10.1002/anie.200460587

32. Gurgel, L. V. A. - "Mercerização e modificação química de celulose e bagaço de cana-de-açúcar com anidrido succínico e trietilenotetramina: preparação de novos materiais quelantes para a adsorção de $\mathrm{Pb}(\mathrm{II}), \mathrm{Cd}(\mathrm{II}), \mathrm{Cr}$ (VI) e Cu (II)", Tese de Doutorado, Universidade Federal de Ouro Preto, Brasil (2007).

33. Gutiérrez, M. C.; De Paoli, M. A. \& Felisberti, M. I. - Compos. Appl. Sci. Manuf., 43, p.1338 (2012). http:// dx.doi.org/10.1016/j.compositesa.2012.03.006

34. Ahankari, S. S.; Mohanty, A. K. \& Misra, M. - Compos. Sci. Technol., 71, p.653 (2011). http://dx.doi.org/10.1016/j. compscitech.2011.01.007

35. Pothan, L. A.; George, J. \& Thomas, S. - Compos. Interface., 9, p.335 (2002). http://dx.doi.org/10.1163/156855402760194692

Enviado: $19 / 06 / 13$ Reenviado: $17 / 09 / 13$ Aceito: $23 / 01 / 14$ 